\title{
Role of Platelet Rich Plasma in Tendinopathy
}

\author{
Shiva C. Acharya ${ }^{1}$, Chintan Sheth ${ }^{2}$ \\ ${ }^{1}$ Consultant, Department of Orthopaedics, Tanish Hospital, Surat, Gujarat, India. \\ ${ }^{2}$ Assistant Professor, Department of Orthopaedics, Adani Medical College, Bhuj-Kutch, Gujarat, India.
}

\section{ABSTRACT}

\section{BACKGROUND}

Tendinopathy is a major problem associated with sports and physical activity in active people over 25 years of age. We wanted to study the effect of PRP in the patients of chronic tendinopathy.

\section{METHODS}

In patients of chronic tendinopathy who failed medical treatment for last 3 months, platelet rich plasma is prepared from patient's own blood. After giving platelet rich plasma patient is advised to take rest for 3 weeks with analgesics. Physiotherapy is started after 3 weeks of injection as this causes pain for first 3 weeks. Patients are advised to join their duty after 3 weeks of injection. All the patients were followed up in OPD at $3^{\text {rd }}$ week, $6^{\text {th }}$ week, 3 months and 6 months. At every follow up, range of motion, visual analogue scale and functional activity score recorded.

\section{RESULTS}

The follow up shows that most of the patients do not get relief within 3 weeks after injection. Follow up shows that 46 patients out of 50 got relief within 6 months after injection. That means $94 \%$ of patients are having relief within 6 months of injection.

\section{CONCLUSIONS}

The findings of this study show that platelet rich plasma injection under ultrasound guidance at the tendon is an effective mode of treatment for patients and takes time but results in gradual decrease in symptoms.

\section{KEY WORDS}

Chronic Tendinopathy, Platelet Rich Plasma
Corresponding Author:

Dr. Shiva Acharya,

A-701, The Evolution,

Opp. Nandini 1-3, B/s Kataria Motors,

Off Vip Road, Surat-395007,

Gujarat. India.

E-mail: dracharyashiv@gmail.com

DOI: $10.14260 /$ jemds/2019/616

Financial or Other Competing Interests: None.

How to Cite This Article:

Acharya SC, Sheth C. Role of platelet rich plasma in tendinopathy. J. Evolution Med. Dent. Sci. 2019;8(37):2836-2840, DOI: 10.14260/jemds/2019/616

Submission 11-03-2019,

Peer Review 30-08-2019,

Acceptance 07-09-2019,

Published 16-09-2019. 


\section{BACKGROUND}

Tendinopathy is a major medical problem. It can be defined as a syndrome of tendon pain, localized tenderness, and swelling that impairs performance. ${ }^{1}$ In chronic tendinopathy thee is an increasing degree of degeneration with little or no inflammation present. The application of PRP has been documented in many fields. ${ }^{2}$ First promoted by M. Ferrari in 1987 as an autologous transfusion component after an open heart operation to avoid homologous blood product transfusion, there are now over 5200 entries in the NCBI for PRP ranging in fields from orthopaedics, sports medicine, dentistry, otolaryngology, neurosurgery, ophthalmology, urology, wound healing, cosmetic, cardiothoracic and maxillofacial surgery. The initial popularity of PRP grew from its promise as a safe and natural alternative to surgery. PRP advocates promoted the procedure as an organically based therapy that enabled healing through the use of one's own natural growth factors. In recent years, scientific research and technology has provided a new perspective on platelets. Studies suggest that platelets contain an abundance of growth factors and cytokines that can affect inflammation, postoperative blood loss, infection, osteogenesis, wound, muscle tear and soft tissue healing. ${ }^{3}$ Research now shows that platelets also release many bioactive proteins responsible for attracting macrophages, mesenchymal stem cells and osteoblasts that not only promote removal of degenerated and necrotic tissue, but also enhance tissue regeneration and healing. Jumper's knee also known as patellar tendonitis characterised by inflammation of patellar tendon. ${ }^{4}$ It is seen in athletes participating in sports like high jump, basket ball, ice hockey, volleyball, rad cycling, soccer, wrestling. ${ }^{5}$ It is seen more in males compare to females and symptoms are often serious resulting in long standing impairment of athletic performance. Different sports specific loading characteristics of the knee extensor apparatus, a younger age and higher body weight seem to be risk factors. ${ }^{6}$

Corticosteroid injections are a commonly administered treatment for tendon disorders. All the usual side effects of corticosteroids are possible such as skin atrophy, skin hypopigmentation, post injection flare of symptoms, infections and possible effects from systemic absorption particularly after multiple injections. There is also possible effect on the mechanical integrity of the tendons themselves. There are several case reports of tendon rupture following corticosteroid injection, particularly involving the Achillis tendon. Ford and DeBender have reported a series of tendon ruptures following the use of corticosteroid injections.

Some animal studies have suggested that local corticosteroid injection may lead to a reduction in tendon strength, but again this finding is not universal. Given possible concerns relating to tendon integrity post- injection, particularly at the Achillis tendon, some argue that the use of intratendinous injections is contraindicated whilst evidence surrounding peritendinous injections is lacking.

Chronic degenerative tendinopathies are frequent and difficult to treat. Tendon healing and regeneration may be improved by injecting autologous growth factors obtained from the patient's blood. Autologous growth factors can be injected with autologous whole blood or platelet-rich plasma. Electronic databases were searched for prospective clinical trials on treatment with autologous growth factors of patients with chronic tendinopathy. Chronic tendinopathy in this study included wrist extensors, flexors, plantar fasciopathy and patellar tendinopathy. There are many proposed treatment options for chronic tendinopathy. Treatment in the form of injections with autologous whole blood or PRP are increasingly used in clinical practice. There are high expectations of these regenerative injections, and there is a clear need for effective conservative therapies. ${ }^{7}$ Many studies showed that injections of autologous growth factors in patients with chronic tendinopathy had a significant impact on improving pain and/or function over time. ${ }^{8}$ PRP injections lead to better midterm clinical results compared with focused shock waves in jumper's knee. ${ }^{9}$ Ultrasound guided PRP injections in chronic Achilles and patellar tendinopathy results in a significant and lasting improvement of clinical symptoms. ${ }^{10,11}$ PRP injections given with ultrasound guidance lead to progressive reduction in the pain and disability compared to dry needling in rotator cuff disease. ${ }^{12}$

Musculoskeletal practitioners began using PRP for tendinopathy in the early 1990s. These early practitioners were primarily trained in the use of prolotherapy. The popularity of PRP grew as physicians began to see clinical results in concentrating a patient's own blood factors.

\section{Objectives of the Study}

- To assess the efficacy of platelet rich plasma in chronic tendinopathies like tennis elbow, golfer's elbow, supraspinatus tendinopathy, patellar tendinopathy, tendo Achilles tendinopathy and plantar fasciitis

- To evaluate the outcomes of this recent modality of treatment for chronic tendinopathies.

- To assess advantages and complications of platelet rich plasma in tendinopathy

- To assess term relief of pain in chronic tendinopathies.

\section{METHODS}

This was a prospective interventional study undertaken in the Department of Orthopaedics, Government Medical College, Surat. Patients with chronic tendinopathy like supraspinatus tendinopathy, patellar tendinopathy, tendo Achilles tendinopathy, planter fasciitis, medial epicondylar tendinopathy, lateral epicondylar tendinopathy came to New Civil Hospital, Surat included in the study after obtaining their informed consent. Diagnostic criteria being pain and tenderness at respective joints, more on movements, X-rays showing sclerosis at greater tuberosity in shoulder, calcaneal spur. In few cases sonography of local part done which showed changes of tendinosis. ${ }^{13}$ This study was undertaken from April 2015 to January 2016.

Clearance from institutional ethical committee was obtained. The patients with no improvement with medical treatment for last 3 months with or without physiotherapy included in the study. A detailed history was obtained for evaluating the mode of trauma, visual analogue score, ${ }^{14}$ chronicity, physiotherapy etc. Detail clinical examination and investigations including complete blood count were carried out before giving injection. Platelet count of blood was checked with that of PRP before giving the injection.

Patients who were fitting into the inclusion criteria and gave consent were enrolled and it came to fifty during the 
time period of this study from April 2015 to January 2016 Sample size though not very big but thought to be reasonable hence total 50 patients were included. The inclusion and exclusion criteria were as follows.

\begin{tabular}{|c|c|}
\hline Range of Visual Analogue Scale & Result \\
\hline$<20$ & Excellent \\
\hline $20-49$ & Good \\
\hline $50-69$ & Fair \\
\hline$>70$ & Poor \\
\hline Table 1. Result as per Visual Analogue Scale \\
\hline
\end{tabular}

Depending on the table shown above, the result is prepared. If Visual Analogue Scale is less than 20 then the result is excellent; if VAS is between 20 to 49 then the result is good; if VAS is between 50 - 69 then the result is fair and if VAS is greater than 70 then the result is poor.

\section{Inclusion Criteria}

Patient with symptoms typical to lateral epicondylar tendinopathy, medial epicondylar tendinopathy, supraspinatus tendinopathy, tendo Achilles tendinopathy, plantar fasciitis and patellar tendinopathy, treated with diclofenac $100 \mathrm{mg}$ twice a day or Ibuprofen $400 \mathrm{mg}$ thrice a day or tramadol $100 \mathrm{mg}$ twice a day and physiotherapy for more than 3 months but not improved.

\section{Exclusion Criteria}

1. Any skin pathology at local site.

2. Symptoms of $<3$ months duration.

3. Patients who have taken chronic anti-platelet therapy for conditions like stroke, myocardial infarction etc.

4. Patients having muscular dystrophy.

5. Patients having more than one chronic tendinopathy.

After getting informed consent platelet rich plasma was prepared from patient's own blood. For which blood drawn and it processed by centrifugation. All procedure done under strict aseptic conditions. PRP hence prepared was injected with in 30 minutes of preparation. After giving injection patient was advised to take rest for 3 weeks. Patients were instructed to apply ice for pain and to avoid using NSAID. Physiotherapy was started after 3 weeks of injection as this was causing pain for first 3 weeks. Patients are advised to join their duty after 3 weeks of injection. All the patients were followed up in OPD at 3 weeks, 6 weeks, 3 months and 6 months. At every follow up, range of motion, visual analogue scale and functional activity score recorded. When the patient has started his/her duty, was recorded. Check for development of any complications.

\section{RESULTS}

The mean age of study group was 38 years for the male. The mean age of study group was 40 years for the female. So it's a low standard deviation which means there was no significant difference between the age of males and females. Fifty percent of males and females belong to $31-40$ years, $70 \%$ of males and females belong to 31-50 years. The chronic tendinopathy was more common in form of tennis elbow and plantar fasciitis. $66 \%$ of patients are having plantar fascitis and tennis elbow. Golfer's elbow was more common in males than females. In these study, patellar tendinopathy was not seen in females.

The follow up shows that most of the patients do not get relief within 3 weeks after injection. The mean of males who got relief within 3 weeks is 0.2 with standard deviation of 0.4 . The mean of females who got relief within 3 weeks is 0 with standard deviation of 0 . Only 1 patient of planter fasciitis got relief from pain at 3 weeks. Pain relief was considered when visual analogue scale of the patient decreased to at least $50 \%$ from pre injection visual analogue scale.

Follow up shows that 16 patients got relief within 6 weeks after injection. Patients with planter fasciitis, tennis elbow, golfer's elbow and patellar tendinopathy seem to get pain relief earlier as compared to patients with supraspinatus and tendo Achilles tendinopathy. At 12 weeks 37 patients out of 50 get pain relief is considered when visual analogue scale of the patient decreased to at least $50 \%$ from pre injection visual analogue score. Result shows that 46 patients out of 50 get relief within 6 months after injection.

\begin{tabular}{|c|c|c|c|c|c|}
\hline VAS & $\begin{array}{c}\text { Pre- } \\
\text { Injection }\end{array}$ & $\begin{array}{c}\text { At 3 } \\
\text { Weeks }\end{array}$ & $\begin{array}{c}\text { At 6 } \\
\text { Weeks }\end{array}$ & $\begin{array}{c}\text { At 12 } \\
\text { Weeks }\end{array}$ & $\begin{array}{c}\text { At 6 } \\
\text { Months }\end{array}$ \\
\hline$\geq 70$ & 46 & 40 & 10 & 4 & 2 \\
\hline $50-69$ & 3 & 7 & 26 & 9 & 3 \\
\hline $20-49$ & 1 & 3 & 14 & 28 & 7 \\
\hline$<20$ & 0 & 0 & 0 & 9 & 38 \\
\hline Total & $\mathbf{5 0}$ & $\mathbf{5 0}$ & $\mathbf{5 0}$ & $\mathbf{5 0}$ & $\mathbf{5 0}$ \\
\hline \multicolumn{6}{|c|}{ Table 2. Distribution of Patients as per VAS } \\
\hline
\end{tabular}

Table 2 shows Visual Analogue Scale (VAS) of patients having chronic tendinopathy. This table shows that pain is gradually decreasing over the time. Within 3 weeks only 10 patients got relief from pain, while within 6 weeks approximately 40 patients got relief. This suggest that Platelet Rich Plasma injection acts gradually over the time. ${ }^{15}$

\begin{tabular}{|c|c|c|c|c|c|}
\hline FAS & $\begin{array}{c}\text { Pre- } \\
\text { Injection }\end{array}$ & $\begin{array}{c}\text { At 3 } \\
\text { Weeks }\end{array}$ & $\begin{array}{c}\text { At 6 } \\
\text { Weeks }\end{array}$ & $\begin{array}{c}\text { At 12 } \\
\text { Weeks }\end{array}$ & $\begin{array}{c}\text { At 6 } \\
\text { Months }\end{array}$ \\
\hline 2 & 46 & 43 & 18 & 6 & 2 \\
\hline 1 & 4 & 7 & 31 & 38 & 16 \\
\hline 0 & 0 & 0 & 1 & 6 & 32 \\
\hline Total & $\mathbf{5 0}$ & $\mathbf{5 0}$ & $\mathbf{5 0}$ & $\mathbf{5 0}$ & $\mathbf{5 0}$ \\
\hline \multicolumn{6}{|c|}{ Table 3. Distribution of Patients as per FAS } \\
\hline
\end{tabular}

Table 3 shows Functional Activity Score (FAS) of patients having chronic tendinopathy. This FAS shows the routine daily activity carried out by a person. Table shows that within 3 weeks there is less improvement compare to 6 weeks. And at the end of 6 months almost all the patients can perform their daily activity smoothly.

Table 4 suggests that quality of platelet count in platelet rich plasma has impact on the result. This table suggest that when the platelet count in platelet rich plasma injection is less than 3.5 times to the baseline level, 33\% patients had fair to poor result. When the platelet count in platelet rich plasma injection is between 3.5 to 5.5 times to the baseline level, significant pain relief found in all patients. When the platelet count in platelet rich plasma injection is more than 5.5 times 
to the baseline level, all the patients have excellent result in chronic tendinopathies.

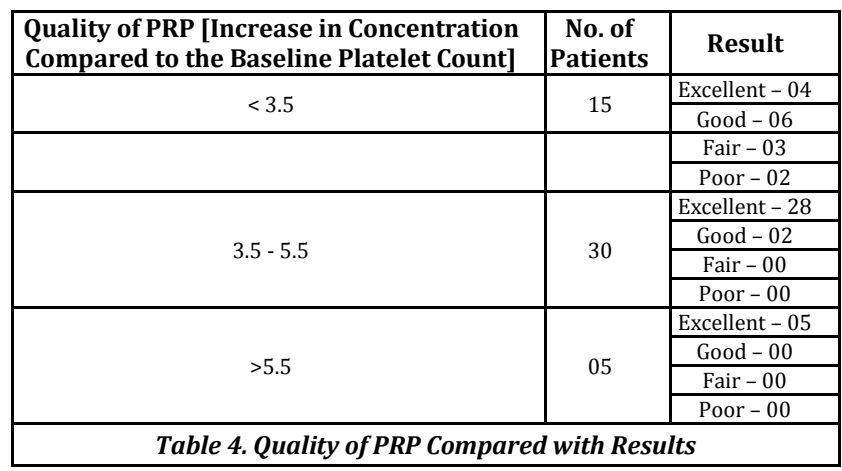

\begin{tabular}{|c|c|c|c|}
\hline Result & Male & Female & Total \\
\hline Excellent & 25 & 12 & 37 \\
\hline Good & 6 & 2 & 8 \\
\hline Fair & 1 & 2 & 3 \\
\hline Poor & 1 & 1 & 2 \\
\hline Total & $\mathbf{3 3}$ & $\mathbf{1 7}$ & $\mathbf{5 0}$ \\
\hline \multicolumn{4}{|c|}{ Table 5. Distribution of Patients as per the Results } \\
\hline
\end{tabular}

Table 5 shows the result of patients after 6 months of treatment. Table shows that $90 \%$ of the patients got excellent to good results with significant relief in pain; $6 \%$ of the total patients had fair result whereas $4 \%$ of the total patients had poor result with minimal relief in pain.

31 male patients were having significant pain relief out of 33 patients and 14 female patients were having significant pain relief out of 17 females. So, in male $94 \%$ of patients having pain relief as compared to female which is $82 \%$. There were no major complications observed in the study apart from local pain at injection site. Also, there was no side effect to patients due to this treatment.

\section{DISCUSSION}

The primary aim of the current study was to determine whether PRP injection would improve clinical parameters i.e., pain and function. Secondary aim included identifying prognostic factors. This study suggests that $82 \%$ of female were having significant pain relief as compared to males which is $94 \%$. This study also suggests that if the platelet count in platelet rich plasma injection is more than 3.5 times to baseline than chance of good result increased. By releasing different platelet growth factors, PRP may be used as a new therapeutic option for chronic tendinopathies. Its ease of preparation, relatively low cost, and minimal invasiveness are arguments in its favour. Furthermore, PRP is not associated with any side effects.

The findings of this study show that platelet rich plasma injection at the tendon is effective mode of treatment for patients with chronic tendinopathies. In this study, mean age of patients having chronic tendinopathy is 38 years in male and 40 years in female. This study shows that $32 \%$ of patients were having improvement in pain and function at 6 weeks of injection and $74 \%$ patients having improvement in pain and function at 12 weeks of injection. $92 \%$ of patients were having significant pain relief at 6 months. This suggest that platelet rich plasma injection takes time to act and this will result in gradual decrease in symptoms. This study also suggest that plantar fasciitis and tennis elbow are more common than other tendinopathies. In this study 32\% patients were having plantar fasciitis and $34 \%$ patients having tennis elbow. This study suggests that $74 \%$ of patients having excellent result in terms of pain and function and $16 \%$ patients having good result in terms of pain and function. Only $4 \%$ of patients having poor result. Platelet rich plasma is very effective in treatment of chronic tendinopathies.

Patients with planter fasciitis, tennis elbow, golfer's elbow and patellar tendinopathy seem to get pain relief earlier as compared to patients with supraspinatus tendinopathy and tendo Achilles tendinopathy. In this study, all the patients with planter fasciitis, golfer's elbow, supraspinatus tendinopathy and patellar tendinopathy have significant pain relief at 6 months as compared to patients with tennis elbow and tendo Achilles tendinopathy in which 4 patients were not having pain relief.

\section{CONCLUSIONS}

PRP may be used as a new therapeutic option for chronic tendinopathies. Its ease of preparation, relatively low cost and minimal invasiveness are arguments in its favour. This suggests that platelet rich plasma injection takes time to act and this will result in gradual decrease in symptoms. One should consider this treatment before going to surgical treatment for chronic tendinopathies. One of the limitations was that the study was carried out with 6 months of follow up, long term results and follow up would have been ideal. PRP with good platelet concentration is associated with enhancement of healing due to its bioactive factors. These are transforming growth factor-beta, platelet derived growth factor. Insulin like growth factor 1 and 2, fibroblast growth factor, vascular growth factor, and endothelial cell growth factor. ${ }^{3}$ The findings of this study show that platelet rich plasma injection under ultrasound guidance at the tendon is effective mode of treating patients with chronic tendinopathies. This suggests that PRP injection takes time to act and this will result in gradual decrease in symptoms. PRP is very effective in treatment of chronic tendinopathies. So, one should consider this treatment before going to surgical treatment for chronic tendinopathies. ${ }^{15}$

\section{REFERENCES}

[1] Jacobs J, Andersson G, Dormans J. Burden of musculoskeletal disease overview. In: The burden of musculoskeletal diseases in the United States: prevalence, societal and economic cost. Rosemont, IL: American Academy of Orthopaedic Surgeons 2011.

[2] Jarvinen TA, Kannus P, Maffulli N, et al. Achilles tendon disorders: etiology and epidemiology. Foot Ankle Clin 2005;10(2):255-66.

[3] Boswell SG, Cole BJ, Sundman EA, et al. Platelet-rich plasma: a milieu of bioactive factors. Arthroscopy 2012;28(3):429-39. 
[4] Blazina ME, Kerlan RK, Jobe FW, et al. Jumper's knee. Orthop Clin North Am 1973;4(3):665-78.

[5] Lian OB, Engebretsen L, Bahr R. Prevalence of jumper's knee among elite athletes from different sports: a crosssectional study. Am J Sports Med 2005;33(4):561-7.

[6] Zwerver J, Bredeweg SW, van den Akker-Scheek I. Prevalence of jumper's knee among nonelite athletes from different sports: a cross-sectional survey. Am J Sports Med 2011;39(9):1984-8.

[7] Kaeding C, Best TM. Tendinosis: pathophysiology and nonoperative treatment. Sports Health 2009;1(4):28492.

[8] Del Buono A, Papalia R, Denaro V, et al. Platelet rich plasma and tendinopathy: state of the art. Int J Immunopathol Pharmacol 2011;24(1 Suppl 2):79-83.

[9] Vetrano M, Castorina A, Vulpiani MC, et al. Platelet-rich plasma versus focused shock waves in the treatment of jumper's knee in athletes. Am J Sports Med 2013;41(4):795-803.

[10] Ferrero G, Fabbro E, Orlandi D, et al. Ultrasound-guided injection of platelet-rich plasma in chronic Achilles and patellar tendinopathy. J Ultrasound 2012;15(4):260-6.
[11] Seo WY, Ha JK, Kim JG, et al. Treatment of chronic patellar tendinitis with platelet rich plasma injection. The Korean Journal of Sports Medicine 2012;30(2):11015.

[12] Rha DW, Park GY, Kim YK, et al. Comparison of the therapeutic effects of ultrasound-guided platelet-rich plasma injection and dry needling in rotator cuff disease: a randomized controlled trial. Clin Rehabil 2013;27(2):113-22.

[13] Khan KM, Bonar F, Desmond PM, et al. Patellar tendinosis (jumper's knee): findings at histopathologic examination, US, and MR imaging. Victorian Institute of Sport Tendon Study Group. Radiology 1996;200(3):8217.

[14] Visentini PJ, Khan KM, Cook JL, et al. The VISA-P score: an index of severity of symptoms in patients with jumper's knee (patellar tendinosis). Journal of Science and Medicine in Sport 1998;1(1):22-8.

[15] Mishra A, Pavelko P. Treatment of chronic elbow tendinosis with buffered platelet rich plasma. The American Journal of Sports Medicine 2006;34(11):17748. 